\title{
Mapping of QTLs controlling root shape and red pigmentation in radish, Raphanus sativus $\mathbf{L}$.
}

\author{
Masato Tsuro*1), Keita Suwabe ${ }^{2,3)}$, Nakao Kubo ${ }^{4,5)}$, Satoru Matsumoto ${ }^{2)}$ and Masashi Hirai ${ }^{4,5)}$ \\ 1) Faculty of Agriculture, Meijo University, Shiogamaguchi, Tenpaku, Nagoya, Aichi 468-8502, Japan \\ 2) National Institute of Vegetable and Tea Science, Ano, Tsu, Mie 514-2392, Japan \\ 3) Present Address: Graduate School of Life Sciences, Tohoku University, Aoba, Sendai, Miyagi 980-8577, Japan \\ 4) Graduate School of Agriculture, Kyoto Prefectural University, Kitainayazuma, Seika, Kyoto 619-0244, Japan \\ 5) Kyoto Prefectural Institute of Agricultural Biotechnology, Kitainayazuma, Seika, Kyoto 619-0244, Japan
}

Genetic factors controlling root shape and red pigmentation on the surface of the hypocotyl (upper part of root) were investigated using a molecular linkage map based on an $F_{2}$ population derived from a cross between two radish cultivars, 'Huang-he hong-wan' and 'Utsugi-gensuke'. One hundred and ninety-eight segregating markers (169 AFLPs, 28 Brassica SSRs and one SLG-CAPS) were located on 14 linkage groups (LGs) at a LOD threshold value of 5.0. The mapping position of Brassica SSRs revealed that frequent genomic rearrangements occurred between the Brassica and Raphanus genomes. Three quantitative trait loci (QTLs) for root shape with LOD values of 2.42, 3.22 and 2.88 were identified on LGs 3, 8 and 9, respectively. These three QTLs accounted for $42.4 \%$ of the phenotypic variance when joined together. Analysis of QTL(s) for root diameter revealed the presence of two QTLs on LG4 and LG8. The QTL on LG8 was considered to control root thickening and to affect the root shape. For hypocotyl pigmentation, a QTL which exerted a large genetic effect with a LOD value of 9.58, was recognized on LG 11 and accounted for $43.8 \%$ of the phenotypic variance.

Key Words: Raphanus sativus, linkage map, QTLs, root shape, red pigmentation.

\section{Introduction}

During the last decade, a number of molecular markers linked to important agronomic traits were developed in many plants (Peleman and van der Voort 2003). Markerassisted selection (MAS) using these molecular markers has accelerated the development of new cultivars and the introduction of desirable traits in breeding programs.

However, many agriculturally important traits are not controlled by a single locus and can involve more than two loci. These loci that underlie a particular trait are designated as quantitative trait loci (QTLs). Genetic linkage map, which is based on molecular markers, is a powerful tool for crop breeding, as it provides a framework for mapping loci that control important traits and enables to gain information about QTLs to be clarified (Paran and Zamir 2003). In addition, comparative mapping analysis of related genomes has been conducted using simple sequence repeat (SSR) and restriction fragment length polymorphism (RFLP) markers to detect the conservation and order of genes, referred to as 'synteny' (Choi et al. 2004, Moore et al. 1995, Suwabe et al. 2006, Tanksley et al. 1992). In cruciferous plants, a large number

Communicated by $\mathrm{T}$. Terachi

Received July 27, 2007. Accepted December 19, 2007.

*Corresponding author (e-mail: tsuro@ccmfs.meijo-u.ac.jp) of reports have dealt with the development of molecular markers linked to important traits and the construction of molecular linkage maps (Ajisaka et al. 2001, Hirai et al. 2004, Kole et al. 1996, Marwede et al. 2005, Mongkolporn et al. 2003, Suwabe et al. 2004b, Tanhuanpää et al. 1996).

Radish, Raphanus sativus L. $(2 \mathrm{n}=2 \mathrm{x}=18)$, is one of the most important vegetables in East Asia, along with other cruciferous crop plants (Kitamura 1958, Kaneko et al. 2007). Its hypertrophied root with hypocotyl is mainly used in cooking, as a pickled vegetable, and in salads. Wide morphological variations have been observed in the roots of lo$\mathrm{cal}$ and commercial radish cultivars. These traits seem to be controlled by QTLs. Some molecular markers linked to important agronomic traits have also been developed in radish. $S$-alleles, which control self-incompatibility, were identified by using the cleaved amplified polymorphic sequence (CAPS) of the $S$-locus-specific glycoprotein (SLG) gene (Niikura and Matsuura 1998). Furthermore, Murayama et al. (2003) screened an amplified fragment length polymorphism (AFLP) marker linked to a fertility restorer $(R f)$ gene for Ogura cytoplasmic male sterility for conversion into a sequence-tagged site (STS) marker. However, these studies were conducted on traits controlled by single dominant genes. To our knowledge, markers for more complex genetic traits have not been studied because whole genome mapping and subsequent QTL analysis would be required. Although 
Bett and Lydiate (2003) reported a linkage map of Raphanus using Brassica RFLP markers, no information about agronomic traits was available from this map.

In a previous study, we constructed a molecular linkage map for an $\mathrm{F}_{1}$ population of radish cultivars based on AFLP, B. rapa genome-derived SSR and SLG markers by adopting a pseudo-test-cross strategy (Tsuro et al. 2005). Although this study provided information about the framework of mapping loci in the $R$. sativus genome, most agronomic traits were not detectable due to the lack of segregation in the $F_{1}$ population. The objective of the present study was to estimate the locations of QTLs controlling the root shape and surface pigmentation of the root and leaf veins in the newly constructed $F_{2}$ linkage map, based on the abovementioned framework markers of the $\mathrm{F}_{1}$ population.

\section{Materials and Methods}

\section{Plant materials}

Two radish cultivars, 'Huang-he hong-wan (Kougabenimaru)' (Sakata Seed Co., Yokohama, Japan) and 'Utsugigensuke' (Ishikawa Agricultural Research Center, Kanazawa, Japan) were used in the present study. 'Huang-he hong-wan' is a Chinese radish cultivar showing a globular root with red skin. 'Utsugi-gensuke' is a Japanese radish with a long white root. Both parental plants used in the present study were the same as those used in the previous study (Tsuro et al. 2005). In each parent, homozygosity was increased by three successive selfings $\left(\mathrm{S}_{3}\right)$. Since outcrossed crucifer plants generally display a high heterology, the $\mathrm{S}_{3}$ plants may still retain some heterozygous loci. However, we consider that such residual heterozygosity did not cause a problem in the present study because the $F_{2}$ population was derived from a single $F_{1}$ plant, in which, for each locus, only one of the alleles should have been transmitted from each parent. One hundred and four $F_{2}$ seeds were germinated on commercial soil ('Ryujo-baido'; Zen-noh Agricultural Co-operatives, Tokyo, Japan) in Jiffy pots placed in a greenhouse in September 2004. After the development of three or four leaves, the seedlings were transferred to the field, planted at $80 \mathrm{~cm}$ intervals in a row $100 \mathrm{~cm}$ wide, and grown under natural conditions. Compound fertilizer $(\mathrm{N}: \mathrm{P}: \mathrm{K}=15: 15: 15)$ at the rate of $180 \mathrm{gm}^{-2}$ was applied as basal dressing. All the plants were harvested at 95 days after seeding, in mid-December 2004.

The root axis of radish is composed of two anatomically distinct parts. The upper part originates from the hypocot$\mathrm{yl}$, namely the stem, and lateral roots do not occur in this part of the root. The lower part consists of true root tissue, and lateral roots are found in this part of the root. These two parts of the radish root become thicker, with the formation of a succulent tissue, which is used as a vegetable for eating. Hereafter, these two parts will be referred to as the 'root' in the present paper. However, since pigmentation on the surface of the two parts of the radish root can differ, the 'root' will be referred to as 'hypocotyl' and 'lower root', respectively. As the roots of the $F_{2}$ plants showed wide variations from slender to hypertrophic like in 'turnip', the ratio of the root length to the diameter at the level of the thickest part of the root was used as an index of root shape. Since the red pigmentation level on the surface of the hypocotyl also showed wide variations in the $F_{2}$ population, we used a visual fivecolor score, namely 0 (white) to 4 (deep red) to indicate increasing pigmentation grade. In addition, the pigmentation of the leaf veins and the surface pigmentation of the lower root were evaluated as red or white, respectively.

\section{DNA polymorphism analysis}

Total DNA was extracted from young leaves of the two parental and $104 \mathrm{~F}_{2}$ plants grown in a greenhouse using the CTAB method (Murray and Thompson 1980). AFLP, Brassica-SSR, and self-incompatibility locus ( $S$-locus) markers were used for the polymorphism analysis. AFLP analysis was conducted according to the method of Vos et al. (1995), with some modifications, as reported previously (Tsuro et al. 2005). Analysis of the SSR polymorphism was carried out using the 'BRMS' primer pairs developed for B. rapa (Suwabe et al. 2002, 2004a). Nineteen SSR loci had already been placed on the genetic linkage maps of $B$. rapa and R. sativus (Suwabe et al. 2006, Tsuro et al. 2005), and eight additional markers are indicated in Table 1. The SSR markers were detected as previously reported (Tsuro et al. 2005). The fragments amplified from BRMS-268 and BRMS-302 were separated by electrophoresis on an $8 \%$ polyacrylamide gel. Polymorphism analysis of SLG was conducted as described previously (Tsuro et al. 2005).

\section{Linkage analysis and QTL detection}

The marker data were scored according to the definition of JoinMap3.0 (Van Ooijen and Voorrips 2001). The $F_{2}$ segregation population was analyzed as an 'F2' population type. The Kosambi mapping function was used for linkage analysis (Kosambi 1944).

For QTL analysis, QTLs for all traits were identified using the Kruskal-Wallis and interval mapping procedure of MapQTL4.0 (Van Ooijen et al. 2002). Significance thresholds for interval mapping were determined using the permutation test of MapQTL $(P<0.05,2500$ permutations). Although interval mapping is widely used, we also applied the Kruskal-Wallis method because in some cases, the residuals obtained with interval mapping were not distributed normally, which might have induced incorrect results (Zini et al. 2005). For the interval mapping approach, five neighboring intervals and a step size of $2 \mathrm{cM}$ were used. A LOD score of 2.0 was adopted to indicate the presence of a QTL.

\section{Results and Discussion}

\section{Linkage mapping and genome rearrangement}

In the AFLP analysis, 280 polymorphic markers were detected using 38 primer pairs and scored as dominant markers. Among the 26 Brassica-SSR primer pairs examined, 16 pairs amplified single polymorphic bands, segregating in a 
Table 1. BRMS markers located on the linkage map of $R$. sativus

\begin{tabular}{|c|c|c|c|c|}
\hline \multirow{2}{*}{ BRMS No. } & \multirow{2}{*}{ Primer sequence $\left(5^{\prime}-3^{\prime}\right)$} & \multirow{2}{*}{$\begin{array}{l}\text { Expected length } \\
\text { in } B . \text { rapa }(\mathrm{bp})^{a}\end{array}$} & \multicolumn{2}{|c|}{ Detected length in R. sativus (bp) } \\
\hline & & & 'Huang-he hong wan' & 'Utsugi-gensuke' \\
\hline \multirow[t]{2}{*}{042} & GGATCAGTTATCTGCACCACAA & 122 & 101 & 99 \\
\hline & TCGGAATTGGATAAGAATTCAA & & & \\
\hline \multirow[t]{2}{*}{089} & GACTTTCCCGATTTGCTTTTTCCAG & 233 & 243 & 245 \\
\hline & GGAGGGTTCGGGTCTTACAAGTTTG & & & \\
\hline \multirow[t]{2}{*}{203} & GAATCAATGGTCCAGATTTCTTTTA & 279 & 328 & 334 \\
\hline & АTCTCTCCCTCTTGTCGTCATCT & & & \\
\hline \multirow[t]{2}{*}{258} & ATCTGATAACTGAGTGTCATTGTGC & 221 & 193 & $\mathrm{ND}^{b}$ \\
\hline & СТCTGCTTCTTCATCGGAAAAAT & & & \\
\hline \multirow[t]{2}{*}{268} & GAGTAGTACCAGGAAGGGTGACCTC & 508 & $\mathrm{ND}^{b}$ & Ca. $100^{c}$ \\
\hline & ACGCTTAACTGCGGAGTTCTGAT & & & \\
\hline \multirow[t]{2}{*}{270} & ACCCAAGTACGTGATGTGAACTTTG & 228 & $\mathrm{ND}^{b}$ & 194 \\
\hline & GAAACAGAAGAGCAGGAGGAGGA & & & \\
\hline \multirow[t]{2}{*}{302} & CAAGTCGAAGAAGACATCTCCAAAC & 331 & Ca. $600^{c}$ & Ca. $580^{c}$ \\
\hline & TAAGCTTCTTCTCGAGCTTTTGGTT & & & \\
\hline \multirow[t]{2}{*}{313} & TCATGGTCACCCGAAGTAATTAAAG & 187 & 188 & 182 \\
\hline & ACCGCAATCTCTCCGATAAACTCTA & & & \\
\hline
\end{tabular}

dominant $(3: 1)$ mode, whereas eight pairs generated codominant polymorphic bands $(1: 2: 1)$. All these primer pairs detected single loci. Two other primer pairs, BRMS303 and BRMS-330, detected two independent loci with two discrete polymorphic bands, respectively. One hundred and ninety-eight segregating markers (169 AFLP, 28 BrassicaSSR and one SLG-CAPS markers) that were used for linkage mapping, generated 14 linkage groups (LGs) at a LOD threshold value of 5.0 (Fig. 1). The total length of the map was $667.6 \mathrm{cM}$, and the length of the LGs ranged from 8.0 (LG14) to $122.2 \mathrm{cM}$ (LG8). The number of markers per LG ranged from 2 (LG7, LG13 and LG14) to 33 (LG1), and the average interval between the markers was $3.4 \mathrm{cM}$. As 111 AFLP markers did not join any linkage group, they remained unmapped. The number of LGs did not match the chromosome number $(n=9)$ of this species. The present map was slightly shorter than reported based on a cross between $R$. sativus and $R$. raphanistrum (Bett and Lydiate 2003). A portion of the genome may have remained unmapped in the present map, although the $R$. sativus and $R$. raphanistrum maps were constructed with Mapmaker, which tends to generate larger maps than those generated with JoinMap (Bradeen et al. 2001).

In a previous study, we constructed a linkage map of $R$. sativus based on an $\mathrm{F}_{1}$ population derived from the same parentage as that in the present analysis (Tsuro et al. 2005). Although the map length in the present study nearly corresponded to that in our previous study $(657.8 \mathrm{cM})$, only seven BRMS loci (BRMS-051, 057-2, 129, 287-1, 303-1, 303-2 and 324) were detected in both populations. The other 12 loci, detected in the $F_{1}$ map, were homozygous, and were not mapped in the $F_{2}$ map. In contrast, 21 BRMS markers, which did not show any segregation in the $F_{1}$ population, were newly located in the present $\mathrm{F}_{2}$ population map. Based on the locations of the SSR and SLG markers, LG1, LG3, LG4 and LG11 in the present $F_{2}$ map could correspond to LG3, LG5, LG1 and LG2 in the previous $\mathrm{F}_{1}$ map (Tsuro et al. 2005), respectively. The correspondence of LG8 in the $F_{2}$ map with an $L G$ in the $F_{1}$ map remained unclear because BRMS-057-2 and BRMS-303-1 were located on LG8 in the present map and on LG3 and LG5 in the previous $\mathrm{F}_{1}$ map, respectively. In another linkage map based on an $\mathrm{F}_{2}$ population derived from the same parental plants as those in the $F_{1}$ map, LG1, LG3 and LG8 in the $F_{1}$ map fully corresponded to three independent linkage groups of the other map, based on the location of SSR markers (data not shown). These results suggested that mapping of BRMS-057-2 at the bottom of LG3 in the previous $F_{1}$ map may have been an artifact due to the lack of connecting markers. LG5 in the previous $F_{1}$ map exhibited two marker clusters, and may have separated when additional markers were used.

In comparative mapping of the SSRs between $B$. rapa (Suwabe et al. 2006) and $R$. sativus (present study), frequent rearrangements might have occurred in the genomes. Thus, each SSR located on the same LG (LG1, LG3, LG4 and LG8) was derived from different Brassica LGs (BLG), respectively. These results corresponded to our previous findings (Tsuro et al. 2005). In cruciferous plants, extensive genomic rearrangements were described based on comparative mapping (see Schranz et al. 2006 for a review). Based on these and our results, the radish genome may have also undergone frequent rearrangements in the evolutionary process, as in other Crucifers. More detailed genomic rearrangements between Brassica and Raphanus genomes could be elucidated by the mapping of a larger number of common markers. 


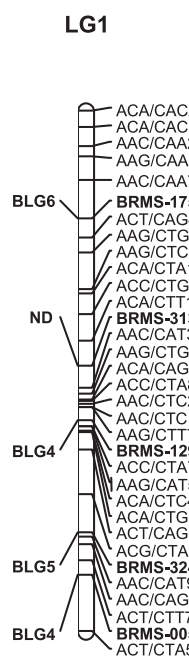

LG9

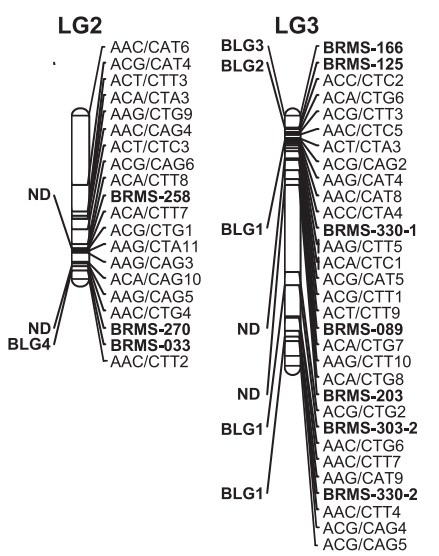

LG10
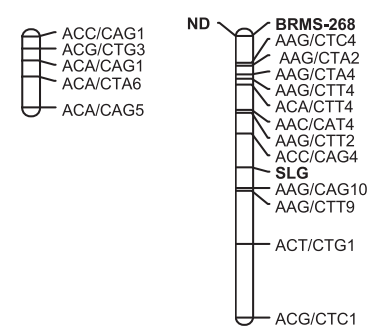

LG4

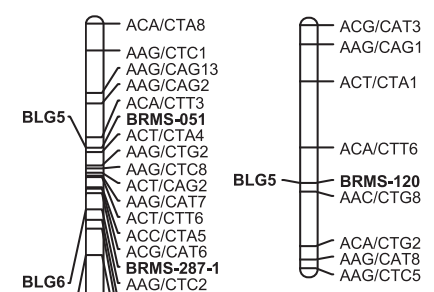

LG6

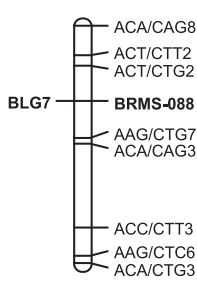

LG7

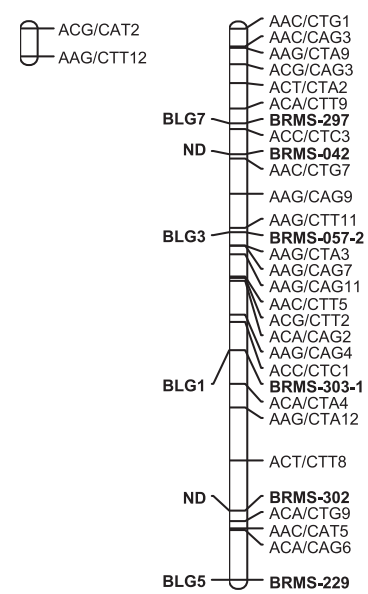

LG12

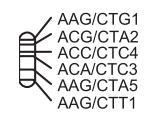

LG13

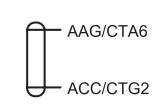

LG14

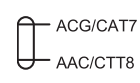

Fig. 1. Linkage map of $R$. sativus based on an $F_{2}$ population derived from a cross between 'Huang-he hong-wan' and 'Utsugi-gensuke' using AFLP, Brassica-SSR and SLG-CAPS markers. SSR and SLG markers are indicated in bold letters. The number identifying each linkage group is shown at the top. The location of the SSR markers on the B. rapa map (Suwabe et al. 2006) is indicated on the left side in each group (e.g. BLG5). SSRs denoted by 'ND' were not located on the B. rapa map.

\section{Root shape}

The value of the root shape index of $\mathrm{S}_{3}$ 'Utsugigensuke', which has a long root, was $3.59 \pm 0.21$, whereas that of $\mathrm{S}_{3}$ 'Huang-he hong-wan', which has a globular root, was $1.09 \pm 0.07$. The value of the root shape index of the $\mathrm{F}_{2}$ individuals showed a continuous normal distribution (Fig. 2A), suggesting that the root shape is controlled by multiple genes. Three QTLs were detected on LG3, LG8 and LG9, respectively. These QTLs exhibited LOD values between 2.42 and 3.22 (Fig. 3A, arrows) and accounted for $42.4 \%$ of the phenotypic variance when joined together (Table 2). These results suggested that the root shape trait is controlled by many QTLs with small effects in addition to the above three QTLs. When the QTL for root length and that for root diameter were separately analyzed, two QTLs controlling the root diameter were detected on LG4 and LG8 (Table 2 and Fig. 3B, arrows). The alleles of 'Huang-he hong-wan' induced an increase of the diameter at both loci, in spite of the small difference in the root diameter of the parents, namely $7.5 \mathrm{~cm}$ for 'Utsugi-gensuke' and $9.6 \mathrm{~cm}$ for 'Huanghe hong-wan'. As expected, since the QTLs for root shape and root diameter were located at the same position on LG8, (Table 2), it was assumed that the QTL on LG8 controlled primarily root thickening and, as a result, affected the root shape. However, the QTL for root length was not detected in the present study, although wide variations in the root length were observed in the parental lines, namely $26.9 \mathrm{~cm}$ for 'Utsugi-gensuke' and $10.3 \mathrm{~cm}$ for 'Huang-he hong-wan', and the segregating $F_{2}$ population. The length of the root may thus be controlled by a number of genes exerting small effects in this population. The present study did not exclude the presence of a QTL for root length with a large effect in Japanese radish cultivars, because the root of the present parent, 'Utsugi-gensuke' was shorter than that of most of the Japanese radish cultivars. Although, the root shape of radish cultivars is known to vary under several environmental conditions, e.g. arcuation and divarication (Iwata et al. 1998), the environmental effect was not as large as to induce a change from long type root to spherical one. We used here a parentage with two distinct phenotypes, namely long root and spherical root. At least in the parental lines, duplicated plants showed similar phenotypes. However, since we used here an $\mathrm{F}_{2}$ population, and evaluated the phenotype of the corresponding genotype based on only one plant, it may be difficult to analyze the environmental effects. Additional data on environmental effects might be obtained by using a recombinant inbred population. 

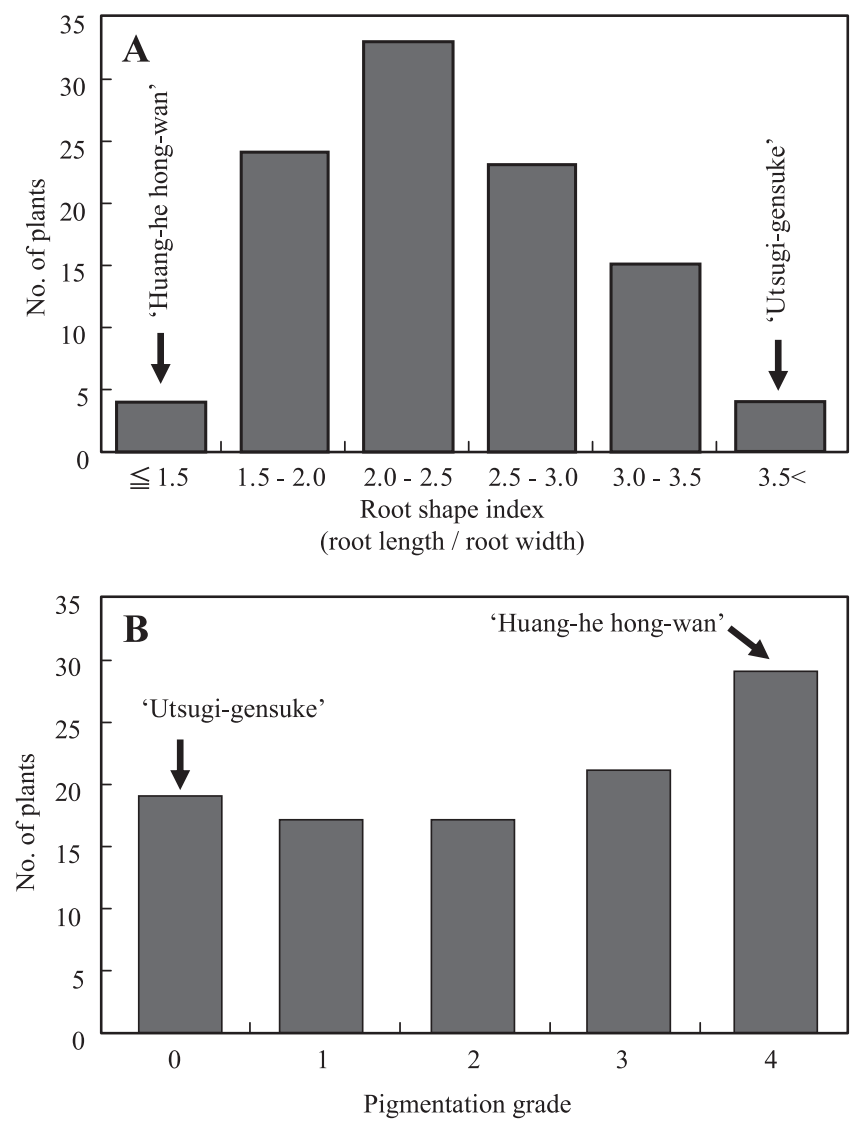

Fig. 2. Frequency distribution of root shape index (A) and red pigmentation of the hypocotyl (B) in an $\mathrm{F}_{2}$ population derived from a cross between 'Huang-he hong-wan' and 'Utsugi-gensuke'. (A) The score for the parental lines was $1.09 \pm 0.07$ for the $S_{3}$ of 'Huang-he hong-wan' $(n=5)$ and $3.59 \pm 0.21$ for the $S_{3}$ of 'Utsugi-gensuke' $(\mathrm{n}=5)$. (B) Pigmentation grade is scored from 0 (white) to 4 (deep red). Scores in the parental lines were 0 for the $S_{3}$ of 'Utsugi-gensuke' $(n=5)$ and 4 for the $S_{3}$ of 'Huang-he hong-wan' $(\mathrm{n}=5)$.

\section{Pigmentation level of root surface and leaf veins}

In all the $F_{1}$ plants, the surface of the hypocotyls showed a deep red pigmentation, while the surface of the lower root was white. The $F_{1}$ plants displayed reddish leaf veins with a gradation from deep red at the base of the vein to green at the tip of the vein. The hypocotyls in 85 out of $104 \mathrm{~F}_{2}$ plants showed various levels of red pigmentation, while in the remaining plants, red pigmentation was not observed. The level of pigmentation in the $\mathrm{F}_{2}$ population displayed a continuous right-biased distribution (Fig. 2B), suggesting that one locus exerted a large effect on pigmentation. Two large peaks in LOD scores were detected on LG11 at positions $29.1 \mathrm{cM}$ and $56.1 \mathrm{cM}$ in interval mapping (Fig. 3C), with only one at $29.1 \mathrm{cM}$, being statistically significant, based on the Kruskal-Wallis test. This QTL exhibited a LOD value of 9.58 and accounted for $43.8 \%$ of the phenotypic variance (Table 2). Genotypic effects on pigmentation were not observed by using the marker closest to the peak at $56.1 \mathrm{cM}$ (ACG/CTC1; data not shown). Since in the present map, only two markers were located in the QTL region at $56.1 \mathrm{cM}$ (Fig. 1), this QTL region requires further analysis.

In the parent, 'Huang-he hong-wan', extensive pigmentation of the leaf veins was observed unlike in the other parent, 'Utsugi-gensuke'. We also examined the segregation of the pigmentation for the two traits: in 92 out of $104 \mathrm{~F}_{2}$ plants, a reddish color of the surface of the leaf veins was observed, while in only six of $104 \mathrm{~F}_{2}$ plants, a red pigmentation of the lower root surface occurred at a very low level. However, the number of loci controlling the pigmentation could not be determined by statistical analysis.

Aoba $(1966,1968)$ reported the presence of an $F_{2}$ segregation of anthocyanin pigmentation for the petiole, hypocotyl and lower root, in several combinations of pigmented or non-pigmented turnips (B. rapa). Nevertheless, the precise number of loci for the pigmentation of the petiole and lower root could not be determined, except for that of the hypocotyl. Based on these and our results, the pigmentation of the leaf veins and lower root of radish may be controlled by complex systems. More detailed analysis on a large scale enable to elucidate the genetic systems involved.

Here, we constructed an $\mathrm{F}_{2}$ genetic linkage map of $R$. sativus, and obtained QTLs for root shape and hypocotyl pigmentation, in addition to some intergenomic information between Raphanus and Brassica. This information might be more useful for Raphanus breeding if the conversion of the present markers to sequence-specific ones could be achieved for saturation of the present map. Further molecular

Table 2. Putative QTLs detected for root shape, thickening and red pigmentation of the hypocotyl in the $\mathrm{F}_{2}$ population of 'Huang-he hong wan' and 'Utsugi-gensuke'

\begin{tabular}{|c|c|c|c|c|c|c|}
\hline Trait & Linkage group & Marker interval $^{a}$ & $\begin{array}{l}\text { QTL position } \\
(\mathrm{cM})\end{array}$ & LOD score & Additive effect ${ }^{c}$ & $\begin{array}{l}\text { Phenotypic variance } \\
\text { accounted for }(\%)\end{array}$ \\
\hline Root shape & 3 & BRMS-303-2-AAC/CTG6 & 39.7 & 2.42 & -0.24 & 11.0 \\
\hline \multirow[t]{2}{*}{ (length/width) } & 8 & AAG/CTA12-ACT/CTT8 & 86.0 & 3.22 & -0.33 & 19.3 \\
\hline & 9 & ACA/CTG5-ACC/CTA6 & 45.4 & 2.88 & 0.25 & 12.1 \\
\hline \multirow[t]{2}{*}{ Thickening } & 4 & ACA/CTT5 ${ }^{b}$ & 77.6 & 3.03 & 0.71 & 13.1 \\
\hline & 8 & AAG/CTA12-ACT/CTT8 & 86.0 & 4.51 & 0.95 & 23.8 \\
\hline Red pigmentation & 11 & SLG-AAG/CAG10 & 29.1 & 9.58 & 1.37 & 43.8 \\
\hline
\end{tabular}

${ }^{a}$ QTL peak position, detected by interval mapping and Kruskal-Wallis analysis (1\%), between two markers.

${ }^{b}$ Terminal side of LG4.

${ }^{c}$ Additive effect of the 'Huang-he hong wan' allele. 
A

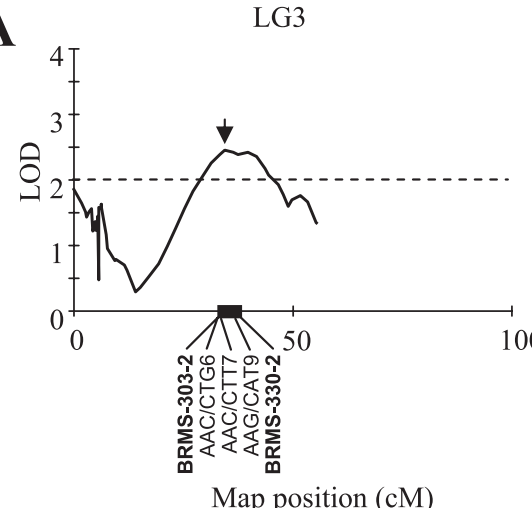

Map position (cM)

B

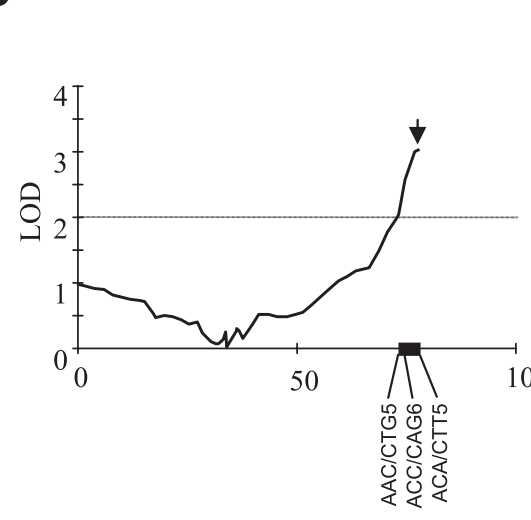

Map position (cM)

C

LG11

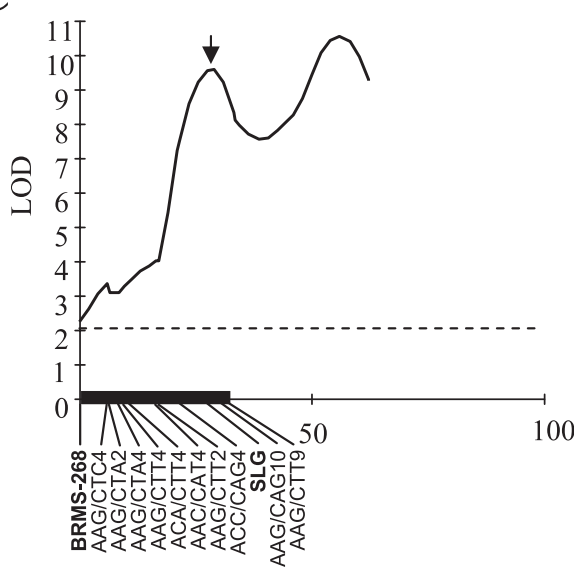

Map position (cM)

Fig. 3. QTL LOD plots for root shape (A), root thickening (B) and pigmentation of the surface of the hypocotyl (C) in R. sativus. The LOD threshold of 2.0 is indicated with a dashed line. Thick line on the horizontal axis indicates statistical significance at the $1 \%$ level based on Kruskal-Wallis analysis. Arrow denotes the peak position of a LOD score in the region, supported by both interval mapping and KruskalWallis analyses.

mapping, including SSR development, is currently being conducted, which could enable to elucidate the genetic factors controlling agronomical traits in $R$. sativus.

\section{Acknowledgements}

The authors thank Ms H. Kasaoka for her technical assistance and the Ishikawa Agricultural Research Center for the supply of 'Utsugi-gensuke' seeds. This work was partly 
supported by Grants-in-Aid from the Ministry of Education, Culture, Sports, Science and Technology of Japan (Nos. 1340006 and 17380006) to M.H.

\section{Literature Cited}

Ajisaka,H., Y.Kuginuki, S.Yui, S.Enomoto and M.Hirai (2001) Identification and mapping of a quantitative trait locus controlling extreme late bolting in Chinese cabbage (Brassica rapa L. ssp. pekinensis syn. campestris L.) using bulked segregant analysis: A QTL controlling extreme late bolting in Chinese cabbage. Euphytica 118: 75-81.

Aoba,T. (1966) Studies on inheritance of root color in turnip (I). J. Yamagata Agr. Forest. Soc. 23: 13-19.

Aoba,T. (1968) Studies on inheritance of root color in turnip (II). J. Yamagata Agr. Forest. Soc. 25: 5-8.

Bett,K.E. and D.J.Lydiate (2003) Genetic analysis and genome mapping in Raphanus. Genome 46: 423-430.

Bradeen,J.M., J.E.Staub, C.Wye, R.Antonise and J.Peleman (2001) Towards an expanded and integrated linkage map of cucumber (Cucumis sativus L.). Genome 44: 111-119.

Choi,H.-K., J.-H.Mun, D.-J.Kim, H.Zhu, J.-M.Baek, J.Mudge, B.Roe, N.Ellis, J.Doyle, G.B.Kiss, N.D.Young and D.R.Cook (2004) Estimating genome conservation between crop and model legume species. Proc. Natl. Acad. Sci. USA 101: 15289-15294.

Hirai,M., T.Harada, N.Kubo, M.Tsukada, K.Suwabe and S.Matsumoto (2004) A novel locus for clubroot resistance in Brassica rapa and its linkage markers. Theor. Appl. Genet. 108: 639-643.

Iwata,H., S.Niikura, S.Matsuura, Y.Takano and Y.Ukai (1998) Evaluation of variation of root shape of Japanese radish (Raphanus sativus L.) based on image analysis using elliptic Fourier descriptors. Euphytica 102: 143-149.

Kaneko,Y., C.Kimizuka-Takagi, S.W.Bang and Y.Matsuzawa (2007) Radish. In "Genome mapping and molecular breeding in plants, Vol. 5 Vegetables" Kole,C. (ed.), Springer-Verlag, Berlin, Heidelberg. p. 141-160.

Kitamura, S. (1958) Cultivars of radish and their change. In "Japanese radish" Nishiyama,I. (ed.), Japan Society for the Promotion of Science, Tokyo. p. 1-19 (in Japanese).

Kole, C., R.Teutonico, A.Mengistu, P.H.Williams and T.C.Osborn (1996) Molecular mapping of a locus controlling resistance to Albugo candida in Brassica rapa. Phytopathology 86: $367-$ 369.

Kosambi,D.D. (1944) The estimation of map distances from recombination values. Ann. Eugenics 12: 172-175.

Marwede,V., M.K.Gül, H.C.Becker and W.Ecke (2005) Mapping of QTL controlling tocopherol content in winter oilseed rape. Plant Breed. 124: 20-26.

Mongkolporn, O., G.P.Kadkol, E.C.K.Pang and P.W.J.Taylor (2003) Identification of RAPD markers linked to recessive genes conferring siliqua shatter resistance in Brassica rapa. Plant Breed. 122: 479-484.

Moore, G., K.M.Devos, Z.Wang and M.D.Gale (1995) Cereal genome evolution: grasses, line up and form a circle. Curr. Biol. 5: 737-739.

Murayama,S., T.Habuchi, H.Yamagishi and T.Terachi (2003) Identification of a sequence-tagged site (STS) marker linked to a restorer gene for Ogura cytoplasmic male sterility in radish
(Raphanus sativus L.) by non-radioactive AFLP analysis. Euphytica 129: 61-68.

Murray,M.G. and W.F.Thompson (1980) Rapid isolation of high molecular weight plant DNA. Nucleic. Acids Res. 8: 4321-4326.

Niikura,S. and S.Matsuura (1998) Identification of self-incompatibility alleles $(S)$ by PCR-RFLP in radish (Raphanus sativus L.). Euphytica 102: 379-384.

Paran,I. and D.Zamir (2003) Quantitative traits in plants: beyond the QTL. Trends Genet. 19: 303-306.

Peleman,J.D. and J.R.vanderVoort (2003) Breeding by design. Trends Plant Sci. 8: 330-334.

Schranz,M.E., M.A.Lysak and T.Mitchell-Olds (2006) The ABC's of comparative genomics in the Brassicaceae: building blocks of crucifer genomes. Trends Plant Sci. 11: 535-542.

Suwabe,K., H.Iketani, T.Nunome, T.Kage and M.Hirai (2002) Isolation and characterization of microsatellites in Brassica rapa $\mathrm{L}$. Theor. Appl. Genet. 104: 1092-1098.

Suwabe,K., H.Iketani, T.Nunome, A.Ohyama, M.Hirai and H.Fukuoka (2004a) Characteristics of microsatellites in Brassica rapa genome and their potential utilization for comparative genomics in Cruciferae. Breed. Sci. 54: 85-90.

Suwabe,K., H.Tsukazaki, H.Ikentani, K.Hatakeyama, M.Fujimura, T.Nunome, H.Fukuoka, S.Matsumoto and M.Hirai (2004b) Identification of two loci for resistance to clubroot (Plasmodiophora brassicae Woronin). Theor. Appl. Genet. 107: 997-1002.

Suwabe, K., H.Tsukazaki, H.Ikentani, K. Hatakeyama, M.Konodo, M.Fujimura, T.Nunome, H.Fukuoka, M.Hirai and S.Matsumoto (2006) Simple sequence repeat-based comparative genomics between Brassica rapa and Arabidopsis thaliana: the genetic origin of clubroot resistance. Genetics 173: 309-319.

Tanhuanpää,P.K., J.P.Vilkki and H.J.Vikki (1996) Mapping a QTL for oleic acid concentration in spring turnip rape (Brassica rapa ssp. oleifera). Theor. Appl. Genet. 92: 952-956.

Tanksley, S.D., M.W. Ganal, J.P.Prince, M.C. de Vicente, M.W. Bonierbale, P.Broun, T.M.Fulton, J.J.Giovannoni, S.Grandillo, G.B.Martin, R.Messeguer, J.C.Miller, L.Miller, A.H.Paterson, O.Pineda, M.S.Röder, R.A.Wing, W.Wu and N.D. Young (1992) High density molecular linkage maps of the tomato and potato genomes. Genetics 132: 1141-1160.

Tsuro, M., K. Suwabe, N.Kubo, S.Matsumoto and M. Hirai (2005) Construction of a molecular linkage map of radish (Raphanus sativus L.), based on AFLP and Brassica-SSR markers. Breed. Sci. 55: 107-111.

Van Ooijen,J.W., M.P.Boer, R.C.Jansen and C.Maliepaard (2002) MapQTL4.0, Software for the calculation of QTL position on genetic maps. Plant Res. Internatl., Wageningen, The Netherlands.

Van Ooijen,J.W. and R.E.Voorrips (2001) JoinMap3.0, Software for the calculation of genetic linkage maps. Plant Res. Internatl., Wageningen, The Netherlands.

Vos,P., R.Hogers, M.Bleeker, M.Reijians, T.van de Lee, M.Hornes, A.Frijters, J.Pot, J.Peleman, M.Kuiper and M.Zabeau (1995) AFLP: a new technique for DNA fingerprinting. Nucleic Acids Res. 23: 4407-4414.

Zini,E., F. Biasioli, F. Gasperi, D. Mott, E. Aprea, T.D. Märk, A. Patocchi, C.Gesseler and M.Komjanc (2005) QTL mapping of volatile compounds in ripe apples detected by proton transfer reaction-mass spectrometry. Euphytica 145: 269-279. 